\title{
Extenuation of Near-Far Effect using Signal Pulsing Schemeand RSIC to reduce MAI in Pseudolite Applications
}

\author{
${ }^{1}$ T. Sruthi, ${ }^{2}$ K.J. Silva Lorraine \\ ${ }^{2}$ Assistant Professor, Dept. of ECE, Sir C.R.Reddy College of Engineering, Eluru, AP, India \\ ${ }^{I}$ Dept. of ECE, Sir C.R.Reddy College of Engineering, Eluru, AP, India
}

\begin{abstract}
The world's most popular GNSS system is Global Positioning System (GPS). This was developed by the U.S Department of Defense (DOD) to know the accurate position coordinates of a location. However, GPS also failed to find the position coordinates especially in dense areas and urban canyon. This situation was eliminated by using ground based satellites called as Pseudolites (PLS) which provide GPS like signals where the obstruction of satellites signals from the GPS constellation take place. Thus, by augmenting the GNSS with Pseudolites the continuity, accuracy and availability were improved. Even though the Pseudolites are useful, still the performance of Pseudolite is limited by the "Near-Far Effect". The various techniques used to overcome near-far Effect are spreading PRN codes, frequency offset, frequency hopping, signal pulsing and Successive Interference Cancellation (SIC). The best way to reduce near-far effect is to use signal pulsing.However, when multiple PLs are employed, multiple access interference limits the system performance. In this paper, the analysis of near-far problem using pulsing scheme has be done while taking GPS constellation into account. Also, the performance evaluation of the PL system with Reference based Successive Interference Cancellation (RSIC) receiver, which is the best optimal method of Multiuser detector (MUD) has been presented.
\end{abstract}

Keywords: GPS, Pseudolite, Near-far Effect, Signal pulsing, RSIC.

\section{Introduction}

The Global Positioning System (GPS) is a global navigation satellite system which was developed by the U.S Department of Defense (DOD) in 1978. The GPS constellation consists of 24 satellites placed at an altitude of approximately $20,000 \mathrm{~km}$ in 6 orbits. Each orbit consists of 4 satellites. By this constellation, at least four and more satellite are visible at the same time from anywhere on the earth. The GPS-based positioning services works on the principle called TRILATERATION which depends on the number of satellites being tracked. The GPS ranging signal consists of ranging information which is encoded by PRN codes and modulated using carrier frequencies. There are two PRN codes for GPS signals which are class of GOLD codes. Those are Coarse and Acquisition (C/A) code, Precise (P) code. GPS operates at L-band frequencies i.e, L1-1575.42MHz, L2-1227.6MHz.

In some areas like urban canyons and deep open cut mines etc., the satellite signals are not being tracked for positioning information. Due to many obstacles the signal strength of the satellite signal decreases. So it can't be tracked by the GPS receiver. So, the positioning coordinates are difficult to calculate by trilateration; because it must have at least 4 satellite signals to know the position. To over-come this problem, ground based satellites called Pseudolites (PLs) which can transmit satellite like signals are used. Pseudolite can be installed wherever required; when placed appropriately, they can provide additional signals to users in locations that obscure signals from GPS satellites. The standard PL transmits signal on $\mathrm{L}_{1}$ frequency and modulates only C/A codes also sends 50bps data message. It uses BPSK modulation. Unlike GPS it uses only low-price TCXO (Temperature compensated crystal oscillator). So, a GPS receiver can track the PL signals too. Even-though PLs are beneficiary, still some problems are associated with various technical issues such as multipath, Near-Far and time synchronization need to be addressed. Among all the problems, the Near-Far problem and Multipath limits the effective dynamic range of truthful positioning. Pulsing the PL signal in the presence of satellite signal has found to be the most competent technique for near-far problem. However, the pulsed PL signals affect GPS receivers depending on its characteristics such as duty cycle and pulse width. But, as the number of PLs increases, the interference called multi access interference will affect the accuracy of position calculations. In this paper, the impact of pulsed PL signal on the GPS receiver while tracking for satellite signal is presented. And the multi access interference reduction using RSIC has also been discussed. 


\section{Near-Far Effect}

The deployment and usage of pseudolites creates a major difficulty in GNSS operation that is near-far operation. This is caused by the higher dynamic range of the signal strength that a receiver experiences when it operates within the range of pseudolite signal transmitters. There are three zones of operation for a pseudolite, namely near region, far region and operating region. For instance, the average signal power of signals coming from GPS L1 C/A satellites is $-130 \mathrm{dBW}$. The pseudolite received power varies as $20 \log _{10}(R)$, where $R$ is the range between the pseudolite and the user's receiving antenna and varies inversely with $\mathrm{R}$. The receiver will be in the operating zone whenever the pseudolite signal power is close to the nominal GPS signal power level and both will be received at the front-end. In the near region, that means when the receiver reaching toward PL the pseudolite,the distance between the PL and receiver decreases gradually. So, signal power is more than that of satellite signal power. So the PL will jam or saturate the satellite signals. So, the receiver will get only the PL signal. In Far region when the receiver moves away from the PL, the distance between PL and the receiver increases gradually. Hence, the satellite signal power will dominate the PL signal power. So the satellite signal will jam or saturate the PL signals and the receiver will receive only the signal from satellite. This problem is called Near-Far effect which is shown in Figure 1.

Thus, Near-Far effect occurs due to the difference in power levels from the satellite and pseudolite at the receiver.$$
P_{r}=P_{t} G_{t} G_{r}\left(\lambda / 4 \pi r^{2}\right)
$$$$
\text { Where, } P_{r} \text { - Received power }
$$$$
P_{t} \text { - Transmitted power }
$$$$
G_{t} \text {.Transmitter antenna gain }
$$ \\ $G_{r}$ - Receiver antenna gain \\ $r$ - Distance between the Transmitter and Receiver}

Equation (1)

The methods to overcome the 'near-far effect' are frequency offset, spreading PRN codes, power control technique and signal pulsing scheme. However, of all these techniques, pulsing scheme have found to be the promising one.

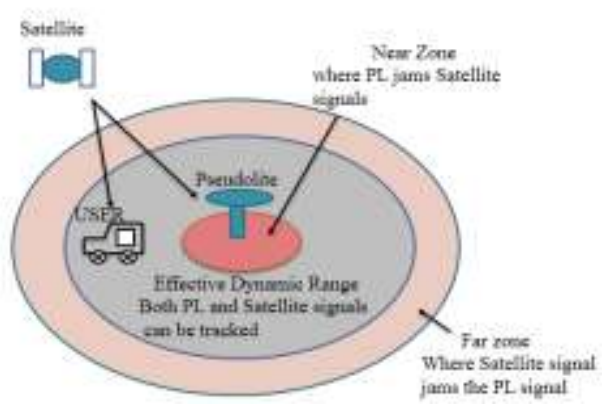

Figure 1: Near-Far scenario

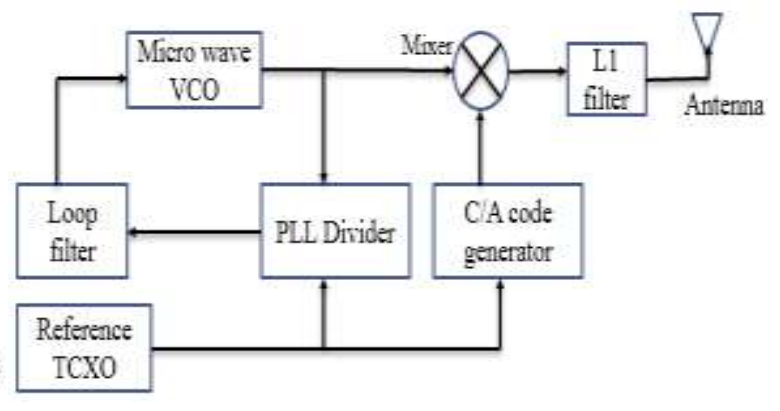

Figure 2: Simple block diagram of Pseudolite

\subsection{Pulsing scheme}

Pulsing is a technique which aims to mitigate against both the near/far and interference problems. The main reason for pulsing is that, a PL signal can interfere with the GPS signal only during the pulses. In this process, the PL signal is broadcasted in short pulses while transmitting nothing in between them. That is, few chips are transmitted at a time and the remaining chips after certain time delay. As a result, the receiver could acquire PL signals from the pulses and satellite. The RTCM committee proposed that the PL signals will transmit frequent, short strong pulses. The pulses are strong enough to be easily tracked, despite having a duty cycle of only $10 \%$ or so. The interval between pulses, with the pseudolite transmitter turned off, would allow receivers to track the satellite signals without interference. 
Required Parameters:

a) Pulse duty Cycle b) Pulse Duration c) Pulse Pattern.

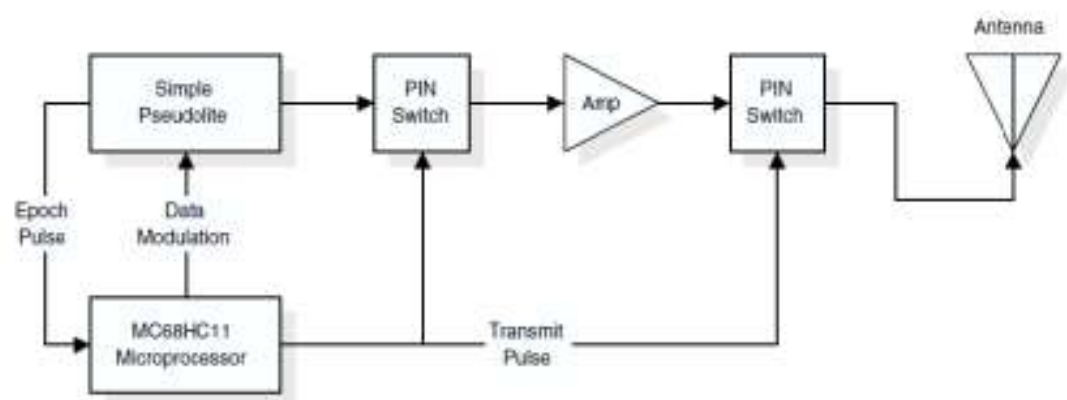

\section{Figure 3: Block diagram of Pulsed Pseudolite}

Types of Pulsing Scheme:

There are two approaches for the design of the pulse sequence

1. Block-based schemes

2. Stream-based schemes.

1.Block-Based Pulsing Schemes: In this, the duration of pulse sequence $T p$ is divided into blocks of $N b c h i p s$. Each block time duration is $T b=N b T c$ seconds. So, the total duration of the pulse sequence is $T p=L b T b$. The chips of each block are divided into $m$ groups of chips, and in each $T b$ seconds only one group of chips is "active". Each group of chips can be identified with a symbol and the set of all $m$ symbols is called the alphabet. The design of a block based pulsing scheme therefore consists of two stages:

1) Design of the sequence of symbols to transmit;

2) Mapping from each symbol to a set of "active" chips.

Permutation sequencesis an important sub-class of the block based schemes. The first complete pulsing scheme was proposed as part of the Radio Technical Commission for Maritime Services (RTCM) specification for DGPS. The RTCM scheme was based on permutation sequences, consisting of 20 full permutations over an alphabet of size $m=10$, and the block size was chosen equal to one full code period. The scheme was applied to the GPS C/A code. Each symbol was mapped to pulses of duration 93 chips. The symbol with index 0 consists of two pulses, the first and last 93 chips in the code. The mapping from symbols to physical pulses illustrated in Figure 4 is precisely the mapping used in the RTCM scheme.

In permutation sequences the duty cycle of the scheme is given as:

$$
d=1 / m \quad \text { where } m \text { is an integer. }
$$

Permutation sequences have many advantages, most notably, in any period of $m T b$ seconds (synchronous with the starting epoch), the entire sequence of symbols is guaranteed to be transmitted for short spreading codes which preserves the correlation properties of codes. Here, $T b$ is equal to the code period. Entire spreading code is transmitted in the minimum time possible for the given duty cycle, without any repetitions of subsequences of the code. For longer codes, this property of permutation sequences is not as important, provided that the receiver coherently integrates the received, pulsed spreading code over a time period that is sufficiently long to ensure a large enough number of chips are received to provide a sharp correlation peak. In this case a long code is defined as one whose period is much longer than the maximum integration time in the receiver [3].

2. Stream-Based Pulsing Schemes:The entire spreading code is treated as a stream of chips, with pulses generated in a pseudorandom fashion synchronous with the spreading code clock. The first stream-based scheme was proposed in 1997. That is Radio Technical Commission for Aeronautics (RTCA) specifications for airport PLs for GPS precision landing guidance [4].

The RTCA choses high speed (10.23 MHz) spreading code with a period of 1 week, based on the GPS Pcode. It was not necessary to build a structured pulsing sequence due to the excellent properties of this spreading code. This scheme was based on the pseudorandom properties of a Linear Feedback Shift Register (LFSR).Figure 5 provides the schematic diagram for the generation of the stream based pulsing scheme.

The design parameters of stream based schemes are

1) Pulse length,

2) Pulse pattern period ( $T p$ ).

The advantage of Stream based is it is easy to implement in both transmitter and receiver side. 


\begin{tabular}{|c|c|c|c|c|c|c|c|c|c|c|}
$d_{0}$ & $d_{1}$ & $d_{2}$ & $d_{3}$ & $d_{4}$ & $d_{5}$ & $d_{6}$ & $d_{7}$ & $d_{8}$ & $d_{9}$ & $d_{10}$ \\
0 & 1 & 2 & 3 & 4 & 5 & 6 & 7 & 8 & 9 & 0 \\
\hline pulse indexes & & & & & & & & & &
\end{tabular}

random index permutation

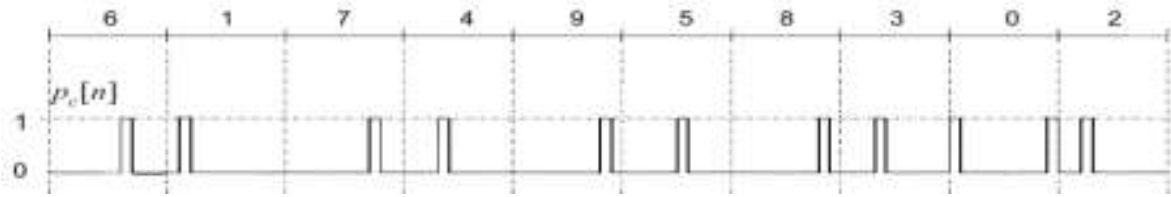

Figure 4: Permutation scheme

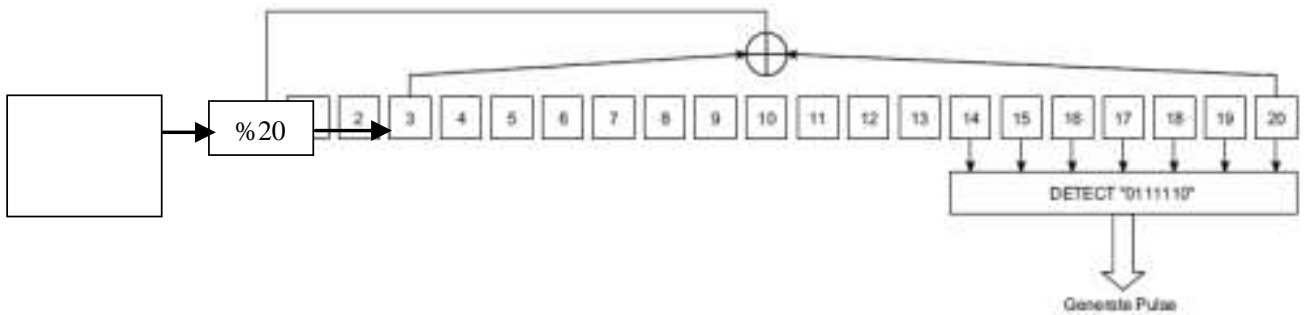

Figure 5: Schematic diagram for pulse generation scheme in RTCM

\section{Multi User Detection}

As the number of PLs increases, multi access interference limits the system performance. A technique which detects desired signal from interference and noise is Multi user detection (MUD). MUD techniques are divided into two types Optimal and Suboptimal detectors [6]. The Conventional Matched Filter (MF) detector was simple to implement, it doesn't require knowledge of the channel or the user amplitudes. Disadvantage of this is it does not take MAI into account and also suffers from near-far problem. Optimum Mud removes the MF detector disadvantages, but it needs knowledge about the channel characteristics at the receiver and this optimal detectors are become complex and grows exponential to the number of users. This can be overcome by suboptimal detector. But, these are infeasible to implement in the real world. So, to reduce complexity in the system, suboptimal technique is being used, it also has better near-far resistance than Matched Filter Detector and also less complex. Again MUD classified into two categories linear and non-linear sub-optimal MUD. The Problemassociated with linear sub-optimal MUD is, it enhances noise and undefined for the situation if there are more users simultaneously using the channel.But, with non-linear sub-optimal MUD complexity does not grow linearly as number of users increases. This is divided into two categories Decision Feedback (DF) and Interference Cancellation (IC). The problem with this technique is same as the linear multi user detector, it requires accurate channel estimates. Due to this complexity, interference cancellation scheme are been sought out as a best detector used at receiver side to improve system capacity. It attempts to remove Multi Access Interference (MAI) after decoding.It successively subtracts the interference from the received signal. The strongest interferer can be detected in the presence of the weaker interferer, and its removal from the received signal enhances the detection of the weaker signals. Thus MAI arises, a technique called Successive interference cancellation (SIC) is a well-known to extract the target signal from multiple signals which causes the unwanted interferences by signal collision or overlapping [6]. SIC uses successive signal subtraction using strong reference signals. Conventional SIC technique is normally applied by one receiver intercepting various signals from multiple transmitters. But, the required signal could be strongly contaminated. So, a new scheme called Referenced-based SIC (RSIC) is proposed.

3.1 Reference- based successive interference cancellation (RSIC) scheme

RSIC successively subtracts interferences by using a relatively clean signal from an initial receiver in a multiplatform receiver system. By developing a receiver in a favored area by initially choosing a target signal which is free of interference, and is referred to as reference signal. This signal can be subtracted in a receiver placed in another area where this signal is considered the only interference (again except for its own receiver noise). Thus the second receiver cancels this interference. The procedure is applicable for the remaining receivers. Therefore, the final target signal can be extracted. By this process the performance of the system is greatly improved. 
There are two different reference-based SIC techniques:

1. Non-Demodulated RSIC

2. Demodulated RSIC.

These two RSIC techniques can be observed in presence of different modulation schemes like QPSK, OFDM.

There are three important variables introduced here. One is the amplitude gain of an interferencesignal denoted by ' $\alpha$ ', its estimate is denoted by ' $\beta$ ', and their difference is given by ' $\gamma$ '. By varying these parameters mainly $\alpha$ and $\gamma$ we will observe the performance of the system towards MAI [7].

\subsubsection{Non- demodulated RSIC (ND-RSIC)}

Let there are four PLs in four targeting areas and four users receiving signals from PLs. The symbol $S_{m}$ represents a signal from PLm, and $n_{n}$ is the additive white Gaussian noise (AWGN) at receiver $n$. $2^{\text {nd }}$ signal can extracted from $2^{\text {nd }}$ Receiver by subtracting $1^{\text {st }}$ signal from signal received at $2^{\text {nd }}$ receiver. An estimate of $1^{\text {st }}$ signalcan be sent from $1^{\text {st }}$ Receiver to $2^{\text {nd }}$ Receiver. That signal is called as reference signal. Repeat this procedure for $3^{\text {rd }}$ signals and $4^{\text {th }}$ signal to extract from $3^{\text {rd }}$ and $4^{\text {th }}$ Receiver by using the subsequent reference signal passed from a prior receiver. In this algorithm, receivers do not need knowledge of the information content. Receiver can simply pass the reference signal to the subsequent receivers for interference cancellation.

In the wireless transmission we have to consider the amplitude loss or gain needs in the model. Here we are using three interference-related variables to model received signals. $\alpha_{m, n}$, is the amplitude gain of unwanted signals from PL mleaking into receiven. $\beta_{m, n}$, is the estimate of $\alpha_{m, n}$ passed from prior receiver $m$ to subsequent receiver $n$. We can write

Received signals

$$
\begin{aligned}
& \gamma_{m, n}=\alpha_{m, n}-\beta_{m, n} \\
& Y_{1}=s_{1}+n_{1} \\
& Y_{2}=s_{2}+\alpha_{1,2} s_{1}+n_{2} \\
& Y_{3}=s_{3}+\alpha_{2,3} S_{2}+\alpha_{1,2} s_{1}+n_{3} \\
& Y_{4}=s_{4}+\alpha_{3,2} S_{3}+\alpha_{2,3} s_{2}+\alpha_{1,2} s_{1}+n_{4}
\end{aligned}
$$

\section{Equation(2)}

If we let $\hat{S}_{1}=\mathrm{S}_{1}+\mathrm{n}_{1}$ be the estimate of desired signal in Receiver 1 , then is $\hat{\mathrm{S}}_{1}$ the initial reference signal and is passed from Receiver 1 to Receiver 2 for cancellation procedure.

Receiver 2 receives the reference signal $\hat{\mathrm{S}}_{1}$, including its own AWGN noise. It performs the reference-

based interference cancellation process as dictated by

$$
\begin{aligned}
& \hat{\mathbf{S}}_{2}=\mathbf{Y}_{2-}-\boldsymbol{\beta}_{1,2} \hat{\mathbf{S}}_{1}+\tilde{\mathbf{n}}_{2} \\
& \quad=\left(\mathbf{s}_{2}+\alpha_{1,2} \mathbf{s}_{1}+\mathbf{n}_{2}\right)-\boldsymbol{\beta}_{1,2}\left(\mathbf{s}_{1}+\mathbf{n}_{1}+\tilde{\mathbf{n}}_{2}\right) \\
& \hat{\mathbf{S}}_{2}=\mathbf{s}_{2}+\left(\boldsymbol{\alpha}_{1,2}-\boldsymbol{\beta}_{1,2}\right) \mathbf{S}_{1}+\mathbf{n}_{2}-\boldsymbol{\beta}_{1,2} \mathbf{n}_{1} \\
& \hat{S}_{2}=\mathbf{s}_{2}+\left(\gamma_{1,2}\right) \mathbf{S}_{1}+\mathbf{n}_{2}-\boldsymbol{\beta}_{1,2} \mathbf{n}_{1}
\end{aligned}
$$

Equation (3)

Similarly, Receiver 3 receives the reference signal $\hat{\mathrm{S}}_{3}$

$$
\hat{\mathbf{S}}_{3}=\mathbf{S}_{3}+\left(\gamma_{2,3}\right) \mathbf{S}_{2}+\left(\gamma_{1,3}-\boldsymbol{\beta}_{2,3} \gamma_{1,2}\right) \mathbf{s}_{1}-\boldsymbol{\beta}_{2,3} \mathbf{n}_{2}+\left(\boldsymbol{\beta}_{2,3} \boldsymbol{\beta}_{1,2}-\boldsymbol{\beta}_{1,3}\right) \mathbf{n}_{1}+\mathbf{n}_{3} \text { Equation (4) }
$$

Receiver 4 receives the reference signal $\hat{\mathrm{S}}_{4}$

$$
\hat{\mathbf{S}}_{4}=\mathbf{S}_{4}+\left(\gamma_{3,4}\right) \mathbf{S}_{3}+\left(\gamma_{2,4}-\boldsymbol{\beta}_{3,4} \gamma_{2,3}\right) \mathbf{S}_{2}-\left[\boldsymbol{\beta}_{3,4}\left(\gamma_{1,3}-\boldsymbol{\beta}_{2,3} \gamma_{1,2}\right)+\boldsymbol{\beta}_{2,4} \gamma_{1,2}\right] \mathbf{S}_{1}-\boldsymbol{\beta}_{3,4} \mathbf{n}_{3}+\left(\boldsymbol{\beta}_{3,4} \boldsymbol{\beta}_{2,3}-\right.
$$

$\left.\boldsymbol{\beta}_{2,4}\right) \mathbf{n}_{2}+\left[\boldsymbol{\beta}_{2,4} \beta_{1,2^{-}}-\beta_{1,4}-\beta_{3,4}\left(\beta_{2,3} \beta_{1,2}-\beta_{1,3}\right)\right] n_{1}+n_{4}$

In this technique, receiver does not require to demodulatethe intercepted signals to perform interference cancellation.

\subsubsection{Demodulated RSIC (D-RSIC)}

In this demodulated scheme, each receiver demodulation is performed.Based on the modulation technique, receivers make decisions (detections) on intercepted signals.

In D-RSIC, the reference signal will be demodulated and re-modulated before transmission to the subsequent receiver. To mitigate noise prior to transmission this additional step is required. Due to this the noise accumulation in the reference signal can be prevented.

Adding the demodulation procedure into Receiver 1, the detection is given by:

$$
\hat{\mathbf{S}}_{1}=\operatorname{dec}\left(\mathbf{s}_{1}+\mathbf{n}_{1}\right)
$$

\section{Equation (6)}

Where, $\quad \hat{\mathbf{S}}_{1}$ is the new initial reference signal which is sent from $1^{\text {st }}$ Receiver to $2^{\text {nd }}$ Receiver. "dec" means decides which symbol is received.

At $2^{\text {nd }}$ Receiver, received reference signal 1 is used for interference cancellation procedure

$$
\hat{\mathbf{S}}_{\mathbf{2}}=\operatorname{dec}\left(\mathbf{y}_{2}-\boldsymbol{\beta}_{1,2} \hat{\mathbf{S}}_{1}+\tilde{\mathbf{n}}_{2}\right)
$$

Equation (7)

In this way the same procedures are repeated at subsequent receivers also. 
IV. Simulation Results

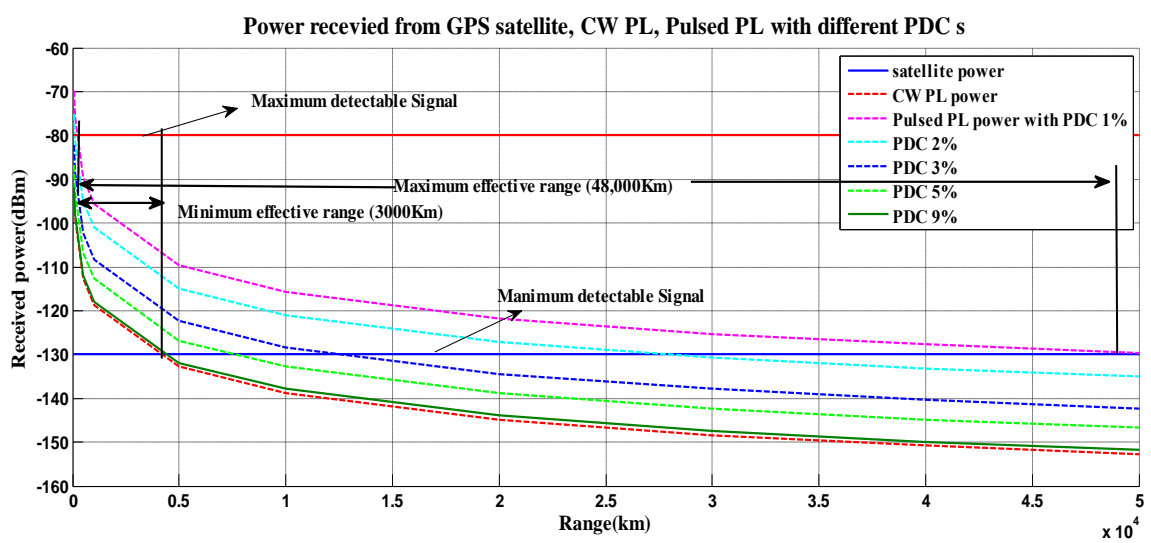

Figure 6: Impact of pulsed PL for various PDC

It can be observed from the figure 6, that the pulsed PL will give more dynamic range than a continuous PL signal. Also, by using PDC of $1 \%$, a dynamic range of $48000 \mathrm{Km}$ can be obtained whereas continuous PL signal provides a range of $3000 \mathrm{~km}$.

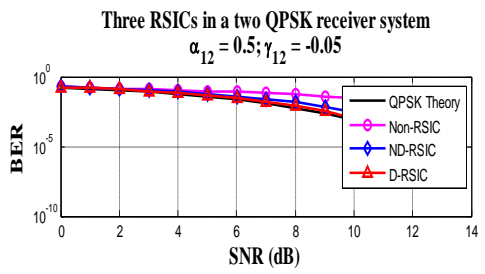

Three RSICs in a two QPSK receiver system $\alpha_{12}=1.5 ; \gamma_{12}=-0.05$

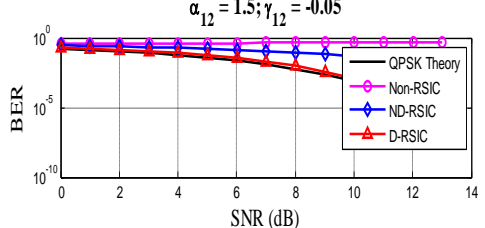

Three RSICs in a two QPSK receiver system $\alpha_{12}=1 ; \gamma_{12}=-0.05$

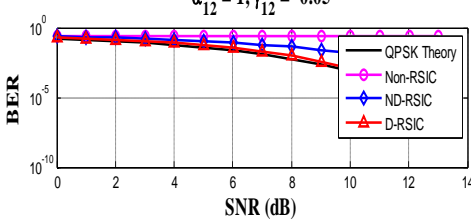

Three RSICs in a two QPSK receiver system $\alpha_{12}=2 ; \gamma_{12}=-0.05$

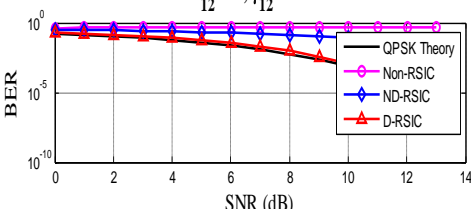

Figure 7: Comparison of SIC and two RISC techniques for QPSK modulation By considering the results of figure7, it can be noticed that D-RSIC reduces the BER much better
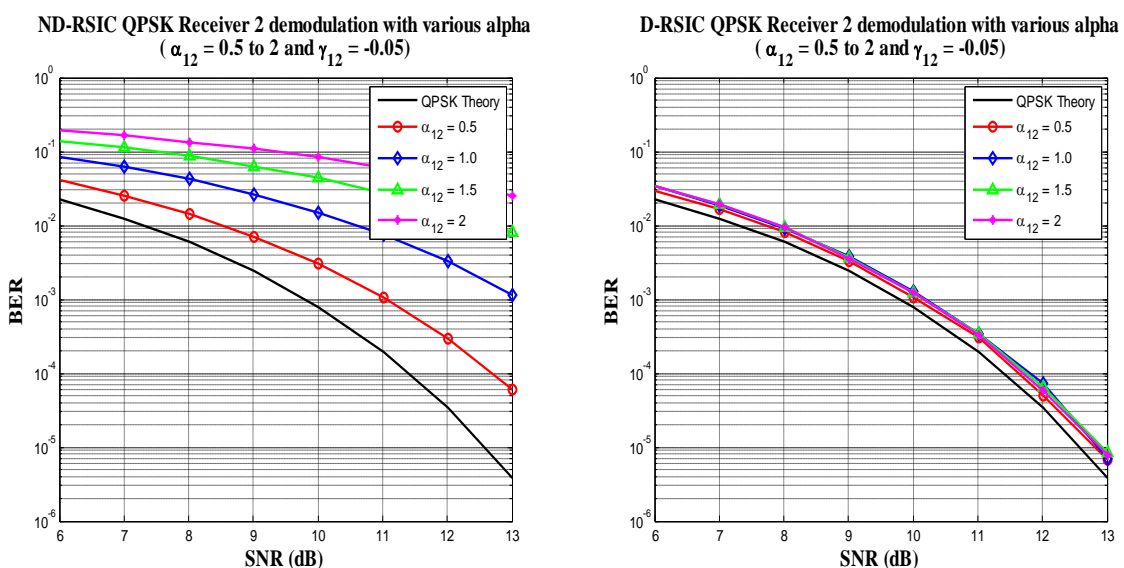

Figure 8: Comparison of ND-RSIC and D-RSIC techniques or QPSK modulation by fixing $\gamma$ valueand by varying $\alpha$ value.

Figure 8 also shows that the D-RSIC is giving best results for less value of $\alpha$. 


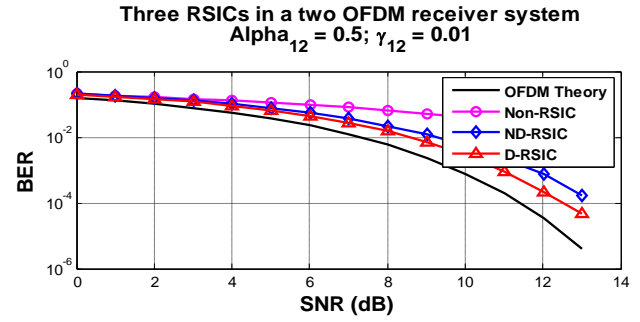

Three RSICs in a two OFDM receiver system

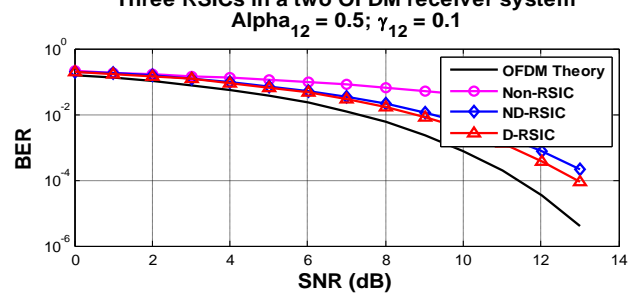

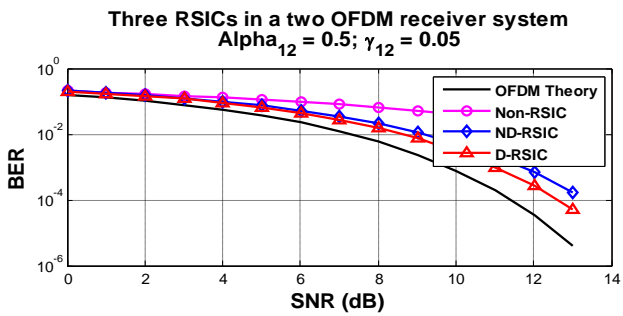

Three RSICs in a two OFDM receiver system

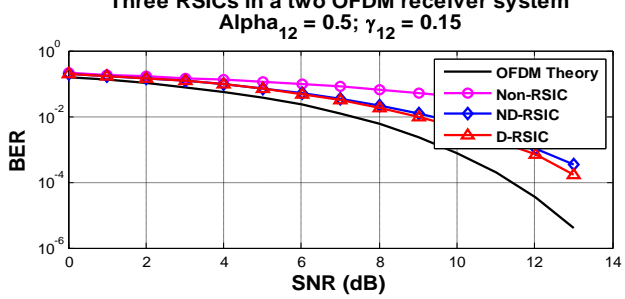

Figure 9: Comparison of results of SIC \& two RSIC techniques with OFDM for fixed $\alpha$, varying $\gamma$.
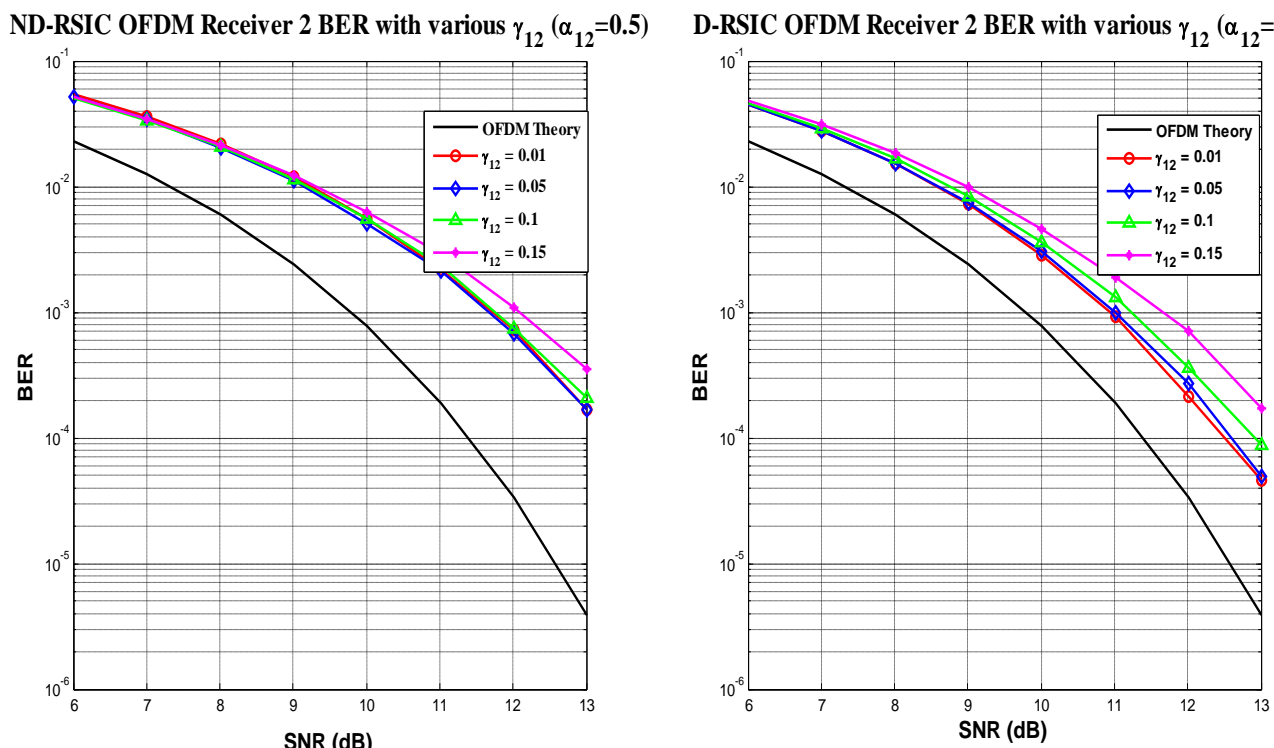

Figure 10: Comparison between ND-RSIC and D-RSIC for OFDM by varying $\gamma$ (Positive value) and constant $\alpha$.

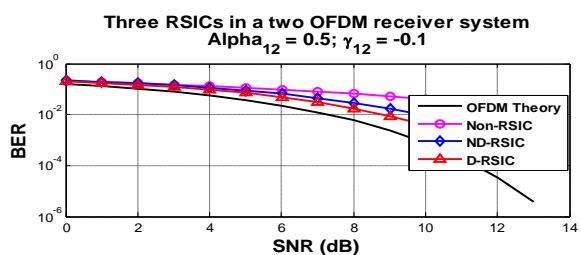

Three RSICs in a two OFDM receiver system

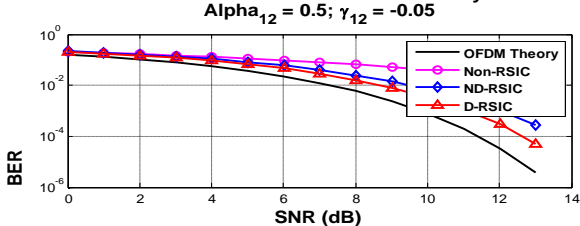

Three RSICs in a two OFDM receiver system
Alpha ${ }_{12}=0.5 ; \gamma_{12}=-0.075$

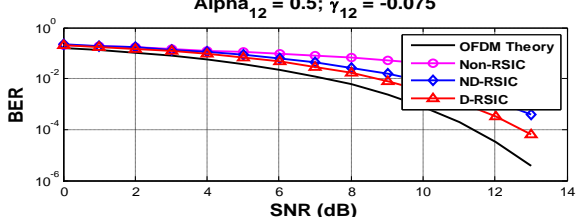

Three RSICs in a two OFDM receiver system

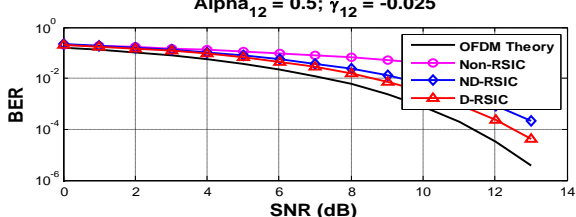

Figure 11: Comparison of results of SIC \& two RSIC techniques with OFDM for fixed $\alpha$, Varyingnegative $\gamma$ value. 

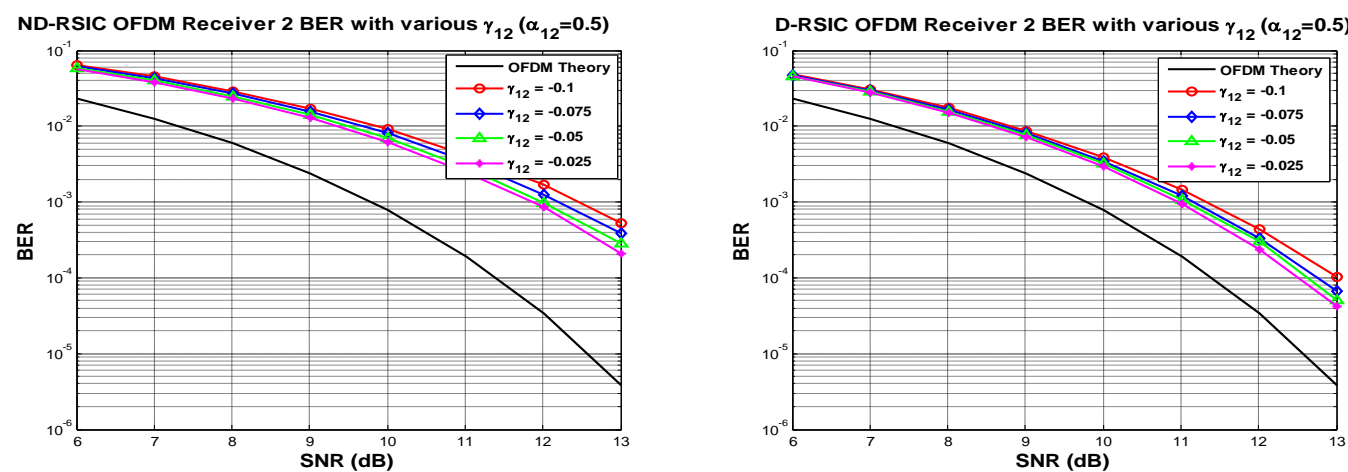

Figure 12: Comparison of results of ND-RSIC, D-RSIC techniques with OFDM for fixed $\alpha$, varyingnegative $\gamma$ value.

By considering figure 9 to 12, it can be concluded that D-RSIC gives less BER than ND-RSIC

Table 1: Comparison between ND-RSIC \& D-RSIC Table 2:Comparison between ND-RSIC \& D-RISC with OFDM for positive $\gamma$ values with OFDM for negative $\gamma$ values

\begin{tabular}{|c|c|c|c|c|c|c|c|c|c|}
\hline$X=12$ & $\underbrace{y}_{\gamma=0.15}$ & $\Gamma=0.1$ & $\gamma=0.05$ & $\gamma=0.01$ & $\mathrm{X}=12$ & у $y=0.1$ & $\gamma=-0.075$ & $\gamma=-0.05$ & $\gamma=-0.025$ \\
\hline $\begin{array}{l}\text { ND- } \\
\text { RSIC }\end{array}$ & 0.00107 & 0.00067 & 0.00074 & 0.0007 & $\begin{array}{l}\text { ND- } \\
\text { RSIC }\end{array}$ & 0.0016 & 0.00126 & 0.0009 & 0.00085 \\
\hline $\begin{array}{l}\text { D- } \\
\text { RSIC }\end{array}$ & 0.0007 & 0.0002 & 0.0003 & 0.0002 & $\begin{array}{l}\text { D- } \\
\text { RSIC }\end{array}$ & 0.00044 & 0.00033 & 0.0003 & 0.000238 \\
\hline
\end{tabular}

Table 3: Comparison between ND-RSIC \&D-RISC Table 4:Comparison between ND-RSIC \& D-RISC with QPSK for positive $\gamma$ values with QPSK for negative $\gamma$ values

\begin{tabular}{|c|c|c|c|c|c|c|c|c|c|}
\hline $\mathrm{X}=12$ & $\begin{array}{l}y=0.01 \\
\gamma\end{array}$ & $\gamma=0.05$ & $\gamma=0.1$ & $\gamma=0.15$ & $X=12$ & $Y=-0.1$ & $\gamma=-0.075$ & $\gamma=-0.015$ & $\gamma=-0.025$ \\
\hline $\begin{array}{l}\text { ND- } \\
\text { RSIC }\end{array}$ & 0.00018 & 0018 & 0.0 & 0 & $\begin{array}{l}\text { ND- } \\
\text { RSIC }\end{array}$ & 0.00056 & 0 & 29 & 021 \\
\hline $\begin{array}{l}\text { D- } \\
\text { RSIC }\end{array}$ & $4 \mathrm{e}-0.05$ & $\begin{array}{l}5.43 \mathrm{e}- \\
0.05\end{array}$ & $\begin{array}{l}9.4 \mathrm{e}- \\
0.05\end{array}$ & $\begin{array}{l}0.0001 \\
7 \\
\end{array}$ & $\begin{array}{l}\text { D- } \\
\text { RSIC }\end{array}$ & 0.0001 & $6.7 \mathrm{e}-0.05$ & $\begin{array}{r}5.325 \mathrm{e}- \\
0.05\end{array}$ & $\begin{array}{r}4.267 \mathrm{e}- \\
0.05\end{array}$ \\
\hline
\end{tabular}

The values for ND-RSIC \& D-RSIC or QPSK and OFDM for different $\gamma$ values are tabulated in Table 1 to table 4 from the simulation results. By observing all the values, it can decided that the D-RSIC with QPSK gives better results of BER.

\section{Conclusion}

Pseudolites are ground-based transmitters that send global navigation satellite system like signals. However, with indoor environment, the positioning method of Pseudolite navigation system is not entirely same as GNSS, and there are some challenging issues in research and system design. In this paper, amajor problem, near-far problem has been analyzedand the dynamic range using pulsing scheme has been presented. By observing the results, it can be concluded that the effective dynamic range will be increased by using pulsed PL signal when compared with the continuous PL signal. Also, the major problem with MAI is performance reduction in means of bit error rate can easily be eliminated by using D-RSIC with QPSK modulation scheme.

\section{References}

[1] H. Stewart cob "GPS Pseudolites: theory design and application", StanfordUniversity, September 1997.

[2] K. J. Silva Lorraine, D.Ajay Kumar, "Analysis of Near-Far Effect and Multipath MitigationTechniques for Pseudolite Based Positioning Applications 'IJECT Vol. 5, Issue Spl - 3, Jan - March 2014

[3] T. L. Abt, F. Soualle, and S. Martin, "Optimal pulsing schemes for Galileo pseudolite signals”Journalof Global Positioning Systems, vol. 6, no. 2, pp. 133-141, 2007.

[4]. CillianO'Driscoll, Daniele Borio “Investigation of Pulsing Schemes for PseudoliteApplications” Conference paper. September 2011.

[5]. Daniele Borio, "Design of a General Pseudolite Pulsing Scheme" IEEE Transactions on aerospace and electronic systems vol. 50, no. 1 January 2014

[6] Madhvi Jangalwa, Sapna Dubey“Performance Improvement of DS-CDMA System with Successive Interference Cancellation Receiver' International Journal of Advanced Research in Computer Science and Software Engineering

[7] Chih-Hwa Ni “Signal Reception via Multi-Platform Receivers”(Major, Republic of China Army.B.S., Chung-Cheng Institute of Technology, 1997) 